\title{
From the journals
}

\section{Medroxyprogesterone may cause loss of bone mineral density}

Results of two clinical trials of medroxyprogesterone for contraception show that women who use intramuscular medroxyprogesterone (Depo-Provera) $150 \mathrm{mg}$ may experience a significant loss in bone mineral density (BMD). The trials have indicated that BMD loss may increase with the duration of use, and may not recover completely after discontinuation of the drug. The drug should be used for the shortest possible time. When it is prescribed the risks and benefits of treatment should be evaluated regularly in all users, particularly in those with a family history of osteoporosis or risk factors for low BMD. Reactions 2005; 1059:3

\section{Insulin glargine: may be responsible for mood changes during treatment}

Two reports have clearly shown that insulin glargine (long acting insulin analogue) was responsible for mood changes of the patients with type 1 diabetes who were on this drug. A 41-year old man with type 1 diabetes developed severe mood swings after switching from human insulin to insulin glargine. His symptoms resolved on discontinuation of the drug. The second report is of a 37-year old man who experienced depressive symptoms when he was switched form pork insulin to insulin glargine. His symptoms too resolved on discontinuation. The WHO Adverse Drug Reactions database also contains two reports of psychosis associated with insulin glargine. Reactions 2005; 1059:15

\section{Non-pharmacological approach for treating head louse infestation}

A recent study has compared the effectiveness of combing and use of a pediculicide for head louse infestation. This study has recruited children and young adults through their general practitioners and by posters and information sheets in local pharmacies and primary schools. For combing, a kit comprising four fine-toothed combs (bug busters) was recommended with instructions to use them with conditioner, four times over 2 weeks. This was compared with a single treatment of a pediculicide available over the counter (malathion or permethrin). The study revealed a cure rate of $57 \%$ with combing versus $13 \%$ with a pediculicide.

Although there are many limitations of this study, such as using a pediculicide once when the recommendation is two applications over 2 weeks, considering the difficulties in the application of a pediculicide it appears that combing is as effective as pediculicide treatment applied twice. The instructions for "bug buster" use is given in the British National Formulary. British Medical Journal 2005; 331: 362-3

\section{Colonoscopic screening compared with flexible sigmoidoscopy}

A study was done in the USA on 1463 women with average risk (asymptomatic and fecal occult blood negative) who were 50 to 79 years of age and referred for colorectal cancer screening at four military medical centres. None had any complications of colonoscopy (e.g. perforation, need for hospitalisation, clinically important bleeding).

Of the 1463 women, 299 had a total of 446 neoplastic lesions. Advanced colorectal neoplasia was present in 72 $(4.9 \%)$ women. Among 230 women with a family history of colon cancer, 16 had advanced neoplasia and 60 had only small adenomas. If only flexible sigmoidoscopy had been performed in all women, advanced colorectal neoplasia would have been identified in only 25 of 1463 women $(1.7 \%)$. There was no significant difference in the diagnostic yield of flexible sigmoidoscopy between women without a family history of colon cancer and women with a family history of colon cancer.A previous study done in men confirmed that some advanced colorectal cancers in men would be missed with the use of flexible sigmoidoscopy but detected by colonoscopy. The present study concludes that colonoscopy may be the preferred method of screening for colorectal cancer in women. New England Journal of Medicine 2005; 352: $2061-8$ 


\section{Guideline for hand hygiene in health care settings}

The new Centers for Disease Control and Prevention guideline provides specific recommendations to promote improved hand hygiene practices. Hand hygiene has been considered to be the most important tool in nosocomial infections control.

The best antimicrobial efficacy can be achieved with ethanol (60-85\%), isopropanol (60-80\%) and $n$-propanol $(60-80 \%)$. Their activity is broad and immediate. The combination of alcohols may have a synergistic antimicrobial effect. The antimicrobial efficacy of chlorhexidine (2-4\%) and triclosan (1-2\%) is both lower and slower. Both agents have a risk of bacterial resistance. Plain soap and water has the lowest efficacy of all. The new guideline promotes alcohol based hand rubs containing various emollients instead of irritating soaps and detergents. Clinical Microbiology Reviews 2004; 17: 863-93.

Kusum de Abrew, Senior Lecturer, Department of Pharmacology, Faculty of Medicine, Colombo. (Competing interests: none declared).

\section{From The Botanic Garden}

Ethereal cohorts! Essences of Air!

Make the green children of the Spring your care!

Oh, Sylphs! disclose in this inquiring age

One Golden Secret to some favoured sage;

Grant the charm'd talisman, the chain, that binds,

Or guides the changeful pinions of the winds!

No more shall hoary Boreas, issuing forth

With Eurus, lead the tempests of the North,

Rime the pale Dawn, or veil'd in flaky showers

Chill the sweet bosoms of the smiling Hours.

By Erasmus Darwin MD, FRS (1731 - 1802), grandfather of Charles Darwin, and a polymath - chemist, botanist, physician, antiquarian. The Botanic Garden is a vast treatise in couplets, its contents ranging from digitalis therapy to the sex life of truffles, and from chemistry to strange plants. Source: "A Primer of English Literature" by Arthur Compton - Rickett MA, LLD; Thomas Nelson and Sons Ltd., London and New York, 1928. 